\title{
VISITA DOMICILIAR COMO ESTRATÉGIA NO MANEJO DA DOR PARA MULHERES COM CÂNCER DE MAMA
}

\author{
*Ana Maria de Almeida, **Marislei Sanches Panobianco, ***Loris Aparecida Prado da Cruz, ***Vanessa \\ Lopes de Aragão, ****Vania Tie Koga Ferreira, *****Maria Antonieta Spinoso Prado
}

\section{RESUMO}

Estudo transversal, descritivo, cujo objetivo foi analisar os registros contidos nos roteiros das visitas domiciliares realizadas pela pesquisa, identificando: as condições de saúde das mulheres visitadas; a existência de metástase e recidiva da doença; a queixa e a localização da dor. Foram identificados 65 prontuários de mulheres cadastradas em um núcleo de reabilitação de mastectomizadas, com 50 visitas realizadas pelo serviço no ano de 2008. Após a coleta, os dados foram organizados no aplicativo Excel da Microsoft e utilizada a estatística descritiva para análise. Foram realizadas 50 visitas domiciliares para 38 mulheres, destas II $(28,9 \%)$ tinham diagnóstico de metástases ou recidiva local. Em 42 (84\%) roteiros das visitas identificou-se algum tipo de queixas de saúde. Destacou-se a queixa emocional presente em $24(48 \%)$ visitas realizadas e a presença de linfedema de braço em 33 visitas (66\%). A queixa de dor foi observada em I7 (34\%) visitas. Conclui-se que a visita domiciliar pode ser uma importante estratégia tanto para as mulheres como para suas famílias no momento em que recebem orientações acerca do autocuidado, manejo da dor e suporte para o enfrentamento da sua condição de saúde.

Palavras-chave: Neoplasias mamárias. Dor. Visita domiciliar.

\section{ABSTRACT}

The aim of the cross-sectional study was to analyze the records contained in the roadmaps of home visits made by identifying: of the women health, the existence of metastasis and recurrence of the disease, the pain complaint and location. There were 65 patient file of women enrolled in a rehabilitation center for mastectomized, with 50 visits made by the department in 2008. After collection, the data were organized in the applicative Microsoft Excel and used the descriptive statistics analysis. It was made fifty home visits to 38 women. Eleven of 38 women $(28.9 \%)$ had a diagnosis of metastasis or local recurrence. In 42 patient file (84\%) was identified some kind of health complaints. In 24 visits $(48 \%)$ was identified the emotional complaint and the presence of arm lymphedema in 33 visits (66\%). The pain complaint was observed in I7 visits (34\%). It was concluded that home visits are an important strategy to both women and their families, and when they receive guidance about self-care, pain management and support for coping with their health condition.

Key words: Breast cancer. Pain. Home visits.

\footnotetext{
* Professora Associada do Departamento de Enfermagem Materno Infantil e Saúde Pública da Escola de Enfermagem de Ribeirão Preto da Universidade de São Paulo (EERP-USP). ** Professora Doutora do Departamento de Enfermagem Materno Infantil e Saúde Pública da EERP-USP. *** Alunas do curso de licenciatura em Enfermagem da EERP-USP e Bolsistas do Fomento às Iniciativas de Cultura e Extensão da Pró-Reitoria de Cultura e Extensão Universitária (PRCEU) da USP. **** Fisioterapeuta, Mestranda do Programa de Pós-Graduação em Enfermagem em Saúde Pública da EERP-USP. ***** Enfermeira, Mestre em Enfermagem e Especialista de Laboratório do Departamento de Enfermagem Materno Infantil e Saúde Pública da EERP-USP. Endereço do autor responsável: Profa. Dra. Ana Maria de Almeida - Escola de Enfermagem de Ribeirão Preto da USP. Av. Bandeirantes, 3900 - Ribeirão Preto-SP - I4040-902 - tel.: (I6) 3602-339I/3432-e-mail: amalmeid@eerp.usp.br
} 


\section{INTRODUÇÃO}

Os avanços tecnológicos e as pesquisas realizadas na área da oncologia têm contribuído para a detecção precoce dos casos de câncer, ao mesmo tempo em que têm possibilitado a descoberta de novos tratamentos. Porém, o câncer ainda representa I3\% dos óbitos mundiais (MENDONÇA et al., 2004).

Mundialmente, o câncer de mama é o quinto de maior mortalidade, tendo atingido 5 I9 mil óbitos em 2004 (WHO, 2007). No Brasil, é a neoplasia maligna que causa maior número de mortes e a segunda de maior incidência entre as mulheres, estimando-se 49 mil novos casos para os anos de 2008 e 2009 (BRASIL, 2008).

Sabe-se que a deteç̧ão precoce do câncer é uma grande arma contra os tratamentos mais agressivos e resultados menos satisfatórios e, no caso do câncer de mama, as medidas eficazes incluem o exame clínico das mamas e da mamografia em conjunto com a ultrassonografia (BRASIL, 2004). Porém, a realidade brasileira ainda mostra grande número de diagnósticos tardios e na atualidade uma maior ocorrência de câncer de mama em mulheres mais jovens, abaixo dos 35 anos.

Internacionalmente, tem-se observado em alguns países desenvolvidos, como é o caso dos Estados Unidos, Canadá, Reino Unido, Holanda, Dinamarca e Noruega, um aumento da incidência do câncer de mama, acompanhado de uma redução da mortalidade, o que está associado à deteç̧ão precoce com o rastreamento e à oferta de tratamento adequado. Em outros países, como no Brasil, o aumento da incidência tem sido acompanhado do aumento da mortalidade, o que pode ser atribuído, principalmente, a um retardamento no diagnóstico e na instituição de terapêutica adequada (SMELTZER \& BARE, 2004).

O estadiamento representa um importante instrumento de avaliação do prognóstico em mulheres com câncer de mama, considerando, em conjunto, as características anatomopatológicas do tumor primário, o comprometimento linfonodal regional e as metástases a distância (NGI, 2004). No Brasil, predomina o estágio III no momento do diagnóstico (BRASIL, I998), o que aumenta significativamente a necessidade de se realizar cirurgias radicais, com retiradas totais da mama, linfonodectomia axilar e complementadas com quimioterapia e radioterapia, o que expõe as mulheres com câncer de mama a complicações futuras, provo- cando um aumento de recidivas e metástases e diminuindo a sua sobrevida.

Entretanto, como é muito difícil precisar como ocorre a progressão da doença, é importante lembrar que as metástases, múltiplas ou únicas, podem aparecer em diversos graus de estadiamento da doença e mesmo antes do diagnóstico da lesão primária (KOKURIAN et al., 2006). Nesta condição da doença metastática, a mulher apresenta comorbidades importantes, a depender do local em que a metástase ocorreu, e pode conviver com estas durante meses e anos de sua vida.

Diante dessas observações, evidencia-se que o câncer de mama não se restringe ao tratamento hospitalar e é de grande importância a implantação do seguimento dessas mulheres, além da reabilitação física e psicossocial com equipe multidisciplinar.

Atendendo a essas necessidades das mulheres com câncer de mama, foi criado o Núcleo de Ensino, Pesquisa e Assistência na Reabilitação de Mastectomizadas (REMA), ligado ao Departamento de Enfermagem Materno Infantil e Saúde Pública da Escola de Enfermagem de Ribeirão Preto da USP. Este núcleo apresenta uma proposta inovadora de extensão de serviços à comunidade, em que articula o ensino de graduação e pós-graduação, presta assistência a mulheres mastectomizadas, às quais se oferece assistência individualizada e/ou coletiva, com vistas à reabilitação física, emocional e social, e desenvolve estudos com o objetivo de compreender aspectos físicos e psicossociais do adoecimento, do cuidado e da reabilitação, identificando novos modelos de assistência, tendo em vista a qualidade do serviço que se reflete na qualidade de vida das mulheres. A assistência prestada fundamentase na própria experiência de vida da mulher, visto que a mulher com câncer de mama não vive, apenas, uma enfermidade biológica, corporal, mas também é, profundamente, afetada psicológica e socialmente, portanto tem como meta a assistência integral (ALMEIDA, 2007; MAMEDE, I99I).

No processo de cuidado, é possível identificar mulheres que enfrentam morbidades relacionadas à evolução da doença, bem como complicações do tratamento: dor, fibrose, linfedema, neuropatia do plexo braquial, diminuição da mobilidade do ombro, fraturas e a recidiva da doença que, muitas vezes, evolui para a morte. Entre as morbidades, destaca-se o quadro de dor, principalmente aquele enfrentado pelas mulheres em estágios avançados da doença. $\mathrm{O}$ enfrentamento da 
dor e a implementação de recursos para seu alívio é um desafio, visto que as mulheres com recorrências e metástases dificilmente apresentam condições físicas para comparecer ao serviço, mas apresentam necessidades de cuidados relacionadas a controle de sintomas, entre eles a dor.

Mulheres com câncer de mama em fase avançada da doença encontram-se, frequentemente, debilitadas e dependentes. A dor, confusão mental, dificuldades respiratórias, alimentares e de locomoção, e a presença de feridas são frequentes, assim como ansiedade e depressão relacionadas a esse processo.

Nessa fase, as mulheres, frequentemente, encontram-se no domicílio, dependentes de cuidados que estão, geralmente, sob a responsabilidade de familiares nem sempre preparados para atendê-las, ou porque desconhecem os procedimentos necessários, ou porque o sofrimento da mulher também afeta os membros da família, promovendo sentimentos relacionados à perda do ente.

Frente a essas questões, o cuidado domiciliar torna-se imprescindível e o REMA tem implantado a visita domiciliar como uma das estratégias para dar seguimento à assistência, principalmente, para as mulheres que estão faltosas no serviço, ou para aquelas em fase avançada da doença, promovendo o suporte para a mulher e família.

Nesse sentido, foi proposto um projeto cujo objetivo foi o de expandir as atividades assistenciais para o âmbito domiciliar, incorporando a família no processo de reabilitação e no cuidado na fase terminal da doença, além de editar um manual que oriente os familiares e/ou cuidadores a implementarem medidas de alívio de sintomas, e a proporcionarem conforto a essas mulheres. O projeto foi aprovado pelo Fomento às Iniciativas de Cultura e Extensão (antigo Fundo de Cultura e Extensão Universitária) da Universidade de São Paulo e as visitas domiciliares foram realizadas por uma enfermeira e alunos de graduação de enfermagem, bolsistas do projeto, às mulheres residentes em Ribeirão Preto.

A visita domiciliar pode ser entendida como atendimento realizado por profissional e/ou equipe de saúde na residência do cliente, com o objetivo de avaliar as suas necessidades, de seus familiares e do ambiente onde vive, para estabelecer um plano assistencial voltado à recuperação e/ou reabilitação (FABRÍCIO et al., 2004).
A assistência no domicílio a pacientes com cuidados paliativos está centrada no binômio pacientecuidador, e para que seja bem sucedida tem que estar amparada pela equipe de saúde. Os cuidadores, sempre que possível e desejando ser informados, devem saber de maneira progressiva e adequada sobre a doença, o tratamento e a indicação e efeitos colaterais mais importantes de cada medicação prescrita. Paciente e familiares devem ser orientados quanto à evolução da doença, quais sintomas e complicações podem ocorrer e o que fazer quando isso acontecer (SAKURADA \& TAQUEMORI, 2008).

Durante a visita, é utilizado um roteiro de avaliação de saúde dessas mulheres, contendo aspectos físicos e emocionais, além de se investigar o motivo pelo afastamento do serviço. Essa experiência da visita domiciliar suscitou questionamentos relacionados às necessidades em saúde, destacando-se o manejo da dor, visto ser este um dos eventos mais frequentes entre as mulheres com câncer de mama.

Frente a esses questionamentos, este estudo teve como objetivos identificar, a partir dos registros dos roteiros de visita domiciliares realizadas no ano de 2008, o perfil sociodemográfico das mulheres visitadas; suas condições de saúde; a presença de metástase e recidiva da doença. Buscou-se, ainda, identificar a ocorrência de dor, sua localização e os recursos utilizados para o seu alívio e controle.

\section{MATERIAL E MÉTODO}

Estudo transversal, descritivo, com abordagem quantitativa, que foi desenvolvido no Núcleo de Ensino, Pesquisa e Assistência na Reabilitação de Mastectomizadas (REMA) da EERP-USP.

O projeto foi aprovado pelo Comitê de Ética em Pesquisa da Escola de Enfermagem de Ribeirão Preto da USP, sob protocolo n. I007/2009.

Foram pesquisados 66 prontuários das mulheres cadastradas no REMA, que haviam sido incluídas nas prioridades de visitas domiciliares, realizadas em cumprimento ao projeto do Fomento às Iniciativas de Cultura e Extensão, no ano de 2008. Nos prontuários, foram analisados os registros de cada roteiro de visita domiciliar, verificando informações sobre: data de nascimento, escolaridade, ocupação, religião, data da cirurgia, condições de saúde que se encontravam as 
mulheres visitadas, presença de metástase e recidiva da doença, a ocorrência de dor, sua localização, e os recursos utilizados para o alívio e controle. Para a coleta de dados, utilizou-se um formulário contendo as variáveis selecionadas.

\section{ANÁLISE DOS DADOS}

Os dados coletados foram organizados no aplicativo Excel da Microsoft e, após a sua consolidação, foi utilizado o programa EpiInfo (versão 2005) para análise estatística descritiva.

\section{RESULTADOS}

Ao longo do ano de 2008 foram agendados 23 dias para realizar as visitas programadas, distribuídas em uma média de 3,39 por dia. Dessas, 28 visitas não foram concluídas pela ausência das mulheres, pelas seguintes situações: duas mulheres tinham ido a óbito, duas não atenderam a equipe, quatro mudaram de endereço, cinco encontravam-se nos serviços de saúde e quinze delas estavam ausentes no momento da visita.

Entre as mulheres visitadas, a maioria $(55,2 \%)$ tinha idade entre 60 a 80 anos, $36,8 \%$ eram casadas, $57,9 \%$ não completaram o ensino fundamental e $57,9 \%$ eram católicas. Em relação ao trabalho, 52,6\% não exerciam atividades remuneradas, isto é, eram donas de casa. O tempo de cirurgia variou de quatro meses a 24 anos, conforme mostra a tabela I.

\section{TABELA 1}

Distribuição das mulheres de um Núcleo de Reabilitação de mastectomizadas, visitadas no ano de 2008, segundo a idade, estado civil, escolaridade, ocupação, religião e tempo de cirurgia. Ribeirão Preto, 2009. $n=38$

\begin{tabular}{|c|c|c|c|}
\hline VARIÁVEIS & CATEGORIAS & $\begin{array}{l}\text { FREQ. } \\
\text { ABSOLUTA }\end{array}$ & $\%$ \\
\hline \multirow{5}{*}{ Idade/ anos } & $43-50$ & 3 & 7,9 \\
\hline & $50-60$ & 7 & I 8,4 \\
\hline & $60-70$ & IO & 26,3 \\
\hline & $70-80$ & II & 28,9 \\
\hline & $80-89$ & 7 & I 8,4 \\
\hline \multirow[t]{4}{*}{ Estado civil } & Solteira & 6 & $\mathrm{I}_{5}, 8$ \\
\hline & Casada & $\mathrm{I} 4$ & 36,8 \\
\hline & Viúva & I3 & 34,2 \\
\hline & Separada & 5 & $\mathrm{I} 3,2$ \\
\hline \multirow[t]{8}{*}{ Escolaridade } & Analfabeta & 4 & IO, 5 \\
\hline & $\begin{array}{l}\text { Ensino fund. } \\
\text { incompleto }\end{array}$ & 22 & 57,9 \\
\hline & $\begin{array}{l}\text { Ensino fund. } \\
\text { completo }\end{array}$ & 3 & 7,9 \\
\hline & $\begin{array}{l}\text { Ensino médio } \\
\text { incompleto }\end{array}$ & I & 2,6 \\
\hline & $\begin{array}{l}\text { Ensino médio } \\
\text { completo }\end{array}$ & 4 & IO, 5 \\
\hline & $\begin{array}{l}\text { Ensino } \\
\text { superior } \\
\text { completo }\end{array}$ & 2 & 5,3 \\
\hline & $\begin{array}{l}\text { Ensino } \\
\text { superior } \\
\text { incompleto }\end{array}$ & I & 2,6 \\
\hline & Não consta & I & 2,6 \\
\hline \multirow[t]{3}{*}{ Ocupação } & $\begin{array}{l}\text { Trabalho não } \\
\text { remunerado }\end{array}$ & 20 & 52,6 \\
\hline & $\begin{array}{l}\text { Trabalho } \\
\text { remunerado }\end{array}$ & I7 & 44,7 \\
\hline & Não trabalha & I & 2,6 \\
\hline \multirow[t]{5}{*}{ Religião } & Católica & 22 & 57,9 \\
\hline & Evangélica & 7 & $\mathrm{I} 8,4$ \\
\hline & Espírita & I & 2,6 \\
\hline & Outra & I & 2,6 \\
\hline & Não consta & 7 & $\mathrm{I} 8,4$ \\
\hline
\end{tabular}




\begin{tabular}{|l|l|l|l|}
\hline $\begin{array}{l}\text { Tempo de } \\
\text { cirurgia } \\
\text { em anos }\end{array}$ & $>2$ & 6 & $\mathrm{I} 5,8$ \\
\cline { 2 - 4 } & 2 a 5 & 8 & $2 \mathrm{I}, \mathrm{I}$ \\
\cline { 2 - 4 } & 5 a IO & IO & 26,3 \\
\cline { 2 - 4 } & IO a I5 & 5 & I3,2 \\
\cline { 2 - 4 } & >I5 & 9 & 23,7 \\
\hline
\end{tabular}

Considerando que um dos critérios adotados pelo núcleo para a programação da visita domiciliar é a condição de saúde das mulheres, no ano de 2008 foram concluídas 50 visitas realizadas para 38 mulheres. Assim, 30 mulheres receberam uma visita, cinco mulheres receberam duas visitas, duas mulheres receberam três visitas, e uma delas recebeu quatro visitas.

Em relação à presença de metástase ou recidivas, identificou-se que II $(28,9 \%)$ delas apresentavam-se nesta condição. Em relação à localização da metástase ou recidiva, estas aconteceram no local cirúrgico em três mulheres $(7,9 \%)$; a metástase óssea foi identificada em quatro mulheres (IO, $5 \%$ ), a pulmonar em três $(7,9 \%)$, na mama contralateral em três $(7,9 \%)$, no cérebro em duas $(5,3 \%)$ e na pele em duas $(5,3 \%)$. Metástases em outros locais também foram identificadas em quatro mulheres (IO, $5 \%$ ) e algumas delas apresentavam metástases em mais de um local.

No registro de 42 (84\%) visitas realizadas, foi identificado algum tipo de queixa de saúde relatado pelas mulheres. É importante salientar que algumas mulheres apresentavam mais de um tipo de queixa, e a referência a aspectos emocionais esteve presente em $24(48 \%)$ registros das visitas realizadas (tabela 2 ).

\section{TABELA 2}

Distribuição dos tipos de queixas identificadas nas visitas realizadas pelo Núcleo de Reabilitação de Mastectomizadas, no ano de 2008. Ribeirão Preto, 2009. n=50

\begin{tabular}{|l|l|l|}
\hline TIPO DE QUEIXAS & FREQ. ABSOLUTA & $\%$ \\
\hline Cardíacas & I7 & 34 \\
\hline Dor & I7 & 34 \\
\hline Emocionais & 24 & 48 \\
\hline Fadiga & I6 & 32 \\
\hline Intestinais & 2 & 4 \\
\hline Locomotora & I8 & 36 \\
\hline Respiratórias & IO & 20 \\
\hline Outras & 27 & 54 \\
\hline
\end{tabular}

As condições do local cirúrgico e do membro homolateral à cirurgia foram avaliadas em cada visita domiciliar e observou-se que em 33 visitas (66\%) foi registrada a presença de linfedema e em sete visitas ( $14 \%$ ) houve o registro da presença de recidiva no local cirúrgico, conforme a tabela 3 .

\section{TABELA 3}

Distribuição das alterações no braço homolateral à cirurgia e local cirúrgico identificadas nas visitas realizadas pelo Núcleo de Reabilitação de Mastectomizadas, no ano de 2008. Ribeirão Preto, 2009. n= 50

\begin{tabular}{|l|l|l|}
\hline VARIÁVEIS & FREQ. ABSOLUTA & $\%$ \\
\hline CONDIÇÕES DO MEMBRO HOMOLATERAL À CIRURGIA \\
\hline Dor & IO & 20 \\
\hline Linfedema & 33 & 66 \\
\hline Fraqueza & 9 & I8 \\
\hline Lesões & I3 & 26 \\
\hline $\begin{array}{l}\text { Sensibilidade } \\
\text { alterada }\end{array}$ & 9 & I8 \\
\hline $\begin{array}{l}\text { Mobilidade } \\
\text { alterada }\end{array}$ & I5 & 30 \\
\hline conDıçÕEs Do LOCAL CIRÚRGICOS & 88 \\
\hline Cicatrização total & 44 & 2 \\
\hline $\begin{array}{l}\text { Deiscência na } \\
\text { cicatriz cirúrgica }\end{array}$ & I & 4 \\
\hline Radiodermite & 2 & \\
\hline
\end{tabular}

Em relação à dor, encontrou-se o registro desta queixa em I7 (34\%) visitas realizadas. Assim, foi possível observar que em $34 \%$ das visitas realizadas no ano de 2008 havia o registro de dor em diferentes localizações e em IO $(20,4 \%)$ a dor foi localizada nos membros superiores. Todas as mulheres visitadas, e que apresentavam queixa de dor, foram orientadas acerca de condutas para o controle da mesma. Destaca-se o fato de que em IO $(58,8 \%)$ visitas o tratamento medicamentoso já era utilizado como a conduta tomada para seu alívio (tabela 4). 


\section{TABELA 4}

Distribuição das queixas de dor, as condutas e indicações identificadas nas visitas realizadas pelo Núcleo de Reabilitação de Mastectomizadas, no ano de 2008. Ribeirão Preto, 2009. n=I7

\begin{tabular}{|c|c|c|}
\hline VARIÁVEIS & FREQ. ABSOLUTA & $\%$ \\
\hline \multicolumn{3}{|l|}{ LOCAL DA DOR } \\
\hline Dor na coluna & 6 & $\mathrm{I} 2,2$ \\
\hline Cabeça & 4 & 8,2 \\
\hline Dor generalizada & 7 & $\mathrm{I} 4,3$ \\
\hline $\begin{array}{l}\text { Dor em membros } \\
\text { inferiores }\end{array}$ & 4 & 8,2 \\
\hline $\begin{array}{l}\text { Dor em membros } \\
\text { superiores }\end{array}$ & IO & 20,4 \\
\hline Quadril & 3 & $6, \mathrm{I}$ \\
\hline \multicolumn{3}{|c|}{ CONDUTAS PARA ALÍVIO DA DOR } \\
\hline $\begin{array}{l}\text { Tratamento } \\
\text { fisioterápico }\end{array}$ & 7 & $4 \mathrm{I}, 2$ \\
\hline $\begin{array}{l}\text { Tratamento } \\
\text { medicamentoso }\end{array}$ & IO & 58,8 \\
\hline Apoio psicológico & 8 & $47, \mathrm{I}$ \\
\hline $\begin{array}{l}\text { Atividades de lazer } \\
\text { e recreação }\end{array}$ & 8 & $47, \mathrm{I}$ \\
\hline
\end{tabular}

\section{DISCUSSÃO}

Nas últimas décadas, observa-se que os avanços nas terapêuticas para o tratamento do câncer de mama têm refletido na qualidade de vida e sobrevida das mulheres diagnosticadas com esta enfermidade.

Há uma série de fatores que afetam a sobrevida de pacientes mastectomizadas, tais como: o estágio em que a doença foi diagnosticada, tipo e tamanho do tumor, recidiva ou metástase da doença e o estilo de vida da paciente. Um dos fatores que influenciam fortemente a sobrevida é a extensão da doença no momento do diagnóstico. O diagnóstico e o tratamento realizados precocemente contribuem significativamente para a sobrevida das pacientes.

No presente estudo, entre as mulheres que foram visitadas, $24(63,2 \%)$ tinham mais que cinco anos de cirurgia, algumas alcançando 24 anos de sobrevida, o que pode apontar para a adequabilidade dos tratamentos implementados. A sobrevida tem sido objeto de estudo na atualidade e pode ser um indicador de que as mulheres têm buscado o tratamento mais precocemente. Assim, a sobrevida de cinco anos é um indicador de avaliação e mostra o sucesso do tratamento planejado que, entre as mulheres estudadas, foi menor que em estudo realizado na Universidade Federal de Santa Maria, no Rio Grande do Sul, que identificou uma sobrevida de cinco anos em $87,7 \%$ das pacientes estudadas (MORAES et al., 2006). Entretanto, há que se considerar que a maioria das mulheres visitadas o foi porque apresentavam sinais de metástases ou recidivas da doença, o que as incluem no grupo que apresentou menor resposta aos tratamentos realizados.

As visitas domiciliares do REMA foram planejadas e realizadas para usuárias faltosas no serviço e para aquelas em fase avançada da doença, com objetivo de manter o vínculo entre usuária e serviço, acompanhar o estado de saúde das mulheres e a evolução da doença, além de oferecer suporte para elas e suas famílias.

Nesse sentido, os roteiros das visitas domiciliares realizadas, entre outros, continham registros sobre recidivas do câncer de mama e metástases. A literatura mostra que a recorrência da doença é maior em pacientes que apresentam comprometimento ganglionar axilar, que aumenta em função do número de linfonodos histologicamente positivos (MENDONÇA et al., 2004). As recidivas locais têm relação estreita com o estado das margens cirúrgicas, a presença de componente intraductal extenso, embolização peritumoral vascular, tipo histológico lobular, grau nuclear e tamanho tumoral. A extensão da ressecção ideal do tecido mamário sofre influência das características do tumor e do tecido mamário circunjacente, modificadas pela idade da paciente, estado hormonal e densidade mamária (RICGI et al., 2003).

Ao se identificar a condição de saúde das mulheres visitadas, verificou-se que as queixas emocionais prevaleceram durante as visitas realizadas, mostrando que a mulher acometida pela câncer de mama, independente do tempo pós-diagnóstico, é afetada emocionalmente.

Concordamos com Rossi \& Santos (2003) sobre a afirmação de que o diagnóstico de câncer confronta o sujeito com a questão do imponderável, da finitude e da morte. Como toda doença potencialmente letal, o câncer induz à perda do corpo saudável, da sensação de invulnerabilidade e de perda de domínio sobre a própria vida e, portanto, torna as mulheres emocionalmente mais vulneráveis. 
A morbidade do membro superior em mulheres submetidas à cirurgia por câncer de mama tem sido estudada, incluindo a dor crônica, alterações de sensibilidade, o linfedema, a limitação dos movimentos do braço, que são os sintomas mais comuns. A severidade de tais sintomas tem grande impacto na realização das atividades diárias e na qualidade de vida das mulheres após o tratamento do câncer de mama (MAGALDI et al. 2005).

Em nosso estudo, o linfedema de braço foi registrado em $66 \%$ das visitas, ressaltando que este dado não se refere ao total de mulheres acometidas pelo linfedema, mas mostra que a sua morbidade é alta e que, uma vez instalado, é de difícil redução, pois as mulheres que foram visitadas mais de uma vez continuavam apresentando-o.

Estudo de revisão de literatura mostrou que a sua prevalência em mulheres submetidas à linfonodectomia axilar para câncer de mama é de 6 a 49\%, dependendo dos critérios adotados para mensuração e definição de linfedema, do tempo transcorrido da cirurgia até a avaliação e das características da população estudada (BERGMANN et al., 2007). Estes achados corroboram com os do nosso estudo.

A técnica da biópsia do linfonodo sentinela foi desenvolvida com o propósito de reduzir o risco de linfedema em mulheres submetidas à cirurgia por câncer de mama. Estudo que comparou a prevalência do linfedema após biópsia do linfonodo sentinela sem linfonodectomia, com biópsia do linfonodo sentinela seguido por linfonodectomia axilar, observou que, mesmo sem linfonodectomia, existe um risco de desenvolver o linfedema, e este risco está associado ao peso corporal, infecção e às lesões que são importantes fatores de risco para desenvolvimento de linfedema (FERREIRA et al., 2008).

Em nosso estudo, foi observado em I3 visitas que as mulheres apresentavam lesões na pele, um dos fatores de risco para desenvolver o linfedema. A visita domiciliar pode ser utilizada como uma das estratégias para educar a mulher ao autocuidado, orientando a preocupação que ela deve ter com o braço.

Outro dado que chama a atenção é que a mobilidade do braço ou a restrição de movimentos do braço homolateral à cirurgia ainda persistia, embora todas tivessem mais de quatro meses de cirurgia. A prática dos exercícios físicos deve ser encorajada, pois são importantes para a melhora da amplitude de movimentos dos braços e ombros (PRADO et al., 2004).

A ocorrência de dor no braço após um ano de cirurgia da mama está mais relacionada à extensão do procedimento cirúrgico axilar do que ao tipo de cirurgia mamária, podendo estar associada a outros fatores como infecção no pós-operatório e alteração de sensibilidade (FERREIRA et al., 2008). Estes dados corroboram com os nossos achados, pois a queixa de dor no braço homolateral à cirurgia foi observada em dez visitas e a sensibilidade do braço alterada em nove visitas.

A dor é uma sensação desagradável, que causa desconforto, podendo variar de leve a insuportável. Pode estar ligada aos diversos aspectos que vão do biológico ao psicológico, e no câncer de mama pode ser proveniente do avanço do tumor ou de consequências do tratamento, como a cirurgia, e pode persistir até mesmo após o tratamento (BRASIL, 200I).

O conceito de dor usado mundialmente hoje é o da Associação Internacional de Estudos da Dor, que afirma que a dor é uma "experiência sensorial e emocional desagradável, associada a dano real ou potencial, ou descrita em termos de tal dano". Significa que a dor é uma experiência única e individual, modificada pelo conhecimento prévio de um dano que pode ser existente ou presumido (ARANTES \& MACIEL, 2008).

Nas visitas realizadas, observamos em I7 roteiros, queixas concretas de dores como cefaleia, dor na coluna, quadril, membros superiores e inferiores e dores generalizadas, porém, se observarmos as condutas para alívio da dor, identificamos em oito roteiros $(47, \mathrm{I} \%)$ que foi oferecido apoio psicológico à mulher, e em oito roteiros $(47, \mathrm{I} \%)$ atividades de lazer e recreação. Esses dados vão ao encontro da literatura que, desde a década de I960, a médica inglesa Cicely Saunders acrescentou ao conhecimento da dor o conceito de dor total, através do qual admite que uma pessoa sofre não apenas pelos danos físicos que possui, mas também pelas consequências emocionais, sociais e espirituais que a proximidade da morte pode lhe proporcionar (ARANTES \& MACIEL, 2008).

Entre os recursos não farmacológicos para o alívio da dor, podemos citar os fisioterápicos, como a drenagem linfática manual, o enfaixamento compressivo funcional, utilizados para evitar ou reduzir o linfedema (MEIRELLES et al., 2006). A estimulação elétrica nervosa transcutânea (TENS) também 
funciona como um analgésico, aumentando a sua tolerância e alívio da dor, a massagem também é um recurso, promove o relaxamento muscular, gerando uma sensação de conforto e alívio da dor (MACIEL \& GÂMARA, 2008).

A dor física é controlada pelo emprego de diferentes analgésicos. Segundo a orientação da Organização Mundial da Saúde, tem se utilizado seis princípios para aliviar a dor do câncer: pela boca; pelo relógio; pela escada; para o indivíduo; uso de adjuvantes; atenção aos detalhes. Conforme uma "escada de três degraus”, na qual em cada degrau há uma substância ou um grupo principal e grupos auxiliares ou adjuvantes. O primeiro degrau é representado pelos analgésicos não opiáceos. Os demais degraus são representados por opiáceos fracos como a codeína, e pelos opiáceos fortes, cujo exemplo é a morfina. Os adjuvantes são os antidepressivos, ansiolíticos, hipnóticos. A dosagem e escolha do analgésico devem ser definidas de acordo com as necessidades de cada indivíduo (BRASIL, 200I).

Outro princípio importante é estar atento aos detalhes do paciente e dos cuidadores que necessitam de instruções precisas, tanto escritas quanto orientadas verbalmente, sobre os nomes dos medicamentos, sua indicação, dosagem, intervalo entre as tomadas e possíveis efeitos colaterais (BRASIL, 200I).

Neste sentido, as condutas para o alívio da dor identificadas nos roteiros das visitas mais uma vez vêm ao encontro com a literatura para tratamento e alívio da dor. Observamos que a utilização de tratamento medicamentoso por orientação médica e a intervenção de outras técnicas não farmacológicas, quer fisioterápicas ou não, como técnicas de distração, lazer e apoio emocional foram estimuladas.

\section{CONCLUSÕES}

Nesse sentido, as visitas domiciliares, além do compromisso de manter o vínculo das mulheres com o serviço de reabilitação e de oferecer apoio nas questões que envolvem a saúde dessas mulheres, principalmente na situação de enfrentamento da recorrência ou em estado de terminalidade, exercem uma importante estratégia, tanto para as mulheres como para suas famílias. É um momento em que recebem orientações quanto ao autocuidado no manejo da dor e suporte para o enfrentamento da sua condição de saúde.
Frente aos resultados encontrados, o grupo responsável pelo projeto desenvolveu um manual de orientação no manejo da dor para mulheres com câncer de mama que será utilizado para orientar as mulheres e seus cuidadores.

\section{REFERÊNCIAS BIBLIOGRÁFICAS}

ALMEIDA, A. M. de. A reabilitação de mulheres com câncer de mama: uma análise descritiva do Núcleo de Ensino, Pesquisa e Assistência na Reabilitação de Mastectomizadas. Ribeirão Preto, 2007. 67p. Tese (Livre Docência). Escola de Enfermagem de Ribeirão Preto, Universidade de São Paulo.

ARANTES, A. G. L. Q; MAGIEL, M. G. S. Avaliação e Tratamento da dor. In: Cuidado Paliativo. Coordenação Institucional de Reinaldo Ayer de Oliveira. São Paulo: Conselho Regional de Medicina do Estado de São Paulo. p. 370-390, 2008.

BERGMANN, A; MATTOS, I. E.; KOIFMAN, R. J. Incidência e prevalência do linfedema após tratamento cirúrgico do câncer de mama. Rev. Bras. Cancerol. v. 53, n. 4, p. 46I-470, out./dez. 2007.

BRASIL, Ministério da Saúde (BR). Instituto Nacional do Câncer. Estimativas de Câncer no Brasil. Rio de Janeiro: INGA, 2008.

BRASIL, Ministério da Saúde (BR). Instituto Nacional do Câncer. Controle do câncer de mama. Documento de Consenso. Rio de Janeiro: INCA, 2004.

BRASIL, Ministério da Saúde. Instituto Nacional do Câncer (INCA). Registros Hospitalares de Câncer (RHC), I998. Instituto Nacional do Câncer. Disponível em: <http://www.inca.gov.br>. Acesso em: I4 dez. 2006.

BRASIL. Ministério da Saúde. Instituto Nacional de Câncer. Cuidados paliativos oncológicos: controle da dor. Rio de Janeiro: INGA, 200 I.

FABRÍCIO, S. G. G.; WEHBE, G.; NASSUR, F. B.; ANDRADE, J. I. Assistência domiciliar: a experiência de um hospital privado do interior paulista. Rev Latinoam Enfermagem. v. I2, n. 5, p. 72I-726, set./out. 2004 .

FERREIRA, B. P. S.; PIMENTEL, M. D.; SANTOS, L.C.dos; DI FLORA, W.; GOBBI, H. Morbidade entre a pós-biópsia de linfonodo sentinela e a dissecção axilar no câncer de mama. Rev. Assoc. Med. Bras. [online]. v. 54, n. 6, p. 5I7-52I, 2008.

KOKURIAN, M.; GARGIA, R.J.; ISHIHARA, H.; PONTE, 
F. M.; VIOLA, D. G. M. Tratamento de metástases do tumor de mama na coluna vertebral. Rev. Bras. Ortop. v. $4 \mathrm{I}, \mathrm{n} .4$, p. II6-I2I, 2006.

MACIEL, A. G. C; CAMARA, S. M. A. Influência da estimulação elétrica nervosa transcutânea (TENS) associada ao alongamento muscular no ganho de flexibilidade. Rev. Bras. Fisioter. v. I2, n. 5, p. 373-378, 2008.

MAGALDI, G. M.; BARROS, A. G. S. D.; MAGALDI, F. M.; MANTESE, J. G.; PINOTTI, J. A. Avaliação da morbidade e funcionalidade do membro superior em mulheres submetidas à linfadenectomia axilar total e biópsia de linfonodo sentinela por câncer de mama. Rev.

Bras. Mastol. n. I, p. 9-I4, 2005.

MAMEDE, M.V. Reabilitação de mastectomizadas: um novo enfoque assistencial. Ribeirão Preto, I99I. I40 p. Tese (Livre Docência). Escola de Enfermagem de Ribeirão Preto, Universidade de São Paulo.

MEIRELLES, M. G. G.; MAMEDE, M. V.; SOUZA, L.; PANOBIANCO, M.S . Avaliação de técnicas fisioterapêuticas no tratamento do linfedema pós-cirurgia de mama em mulheres. Rev. Bras. Fisioter. v. IO, n. 4, p. 393-399, 2006.

MENDONÇA, G. A. S.; SILVA, A. M.; GAUlA, W. M. Características tumorais e sobrevida de cinco anos em pacientes com câncer de mama admitidas no Instituto Nacional de Câncer, Rio de Janeiro, Brasil. Cad. Saúde Púb. v. 20, n. 5, 2004.

MORAES, A. B. de; ZANINI, R. R.; TURCHIELLO, M. S.; RIBOLDI, J.; MEDEIROS, L. R. de. Estudo da sobrevida de pacientes com câncer de mama atendidas no hospital da Universidade Federal de Santa Maria, Rio Grande do Sul, Brasil. Cad. Saúde Púb. v. 22, n. IO, p. 2219-2228, out. 2006.

NATIONAL CANGER INSTITUTE, 2004. Disponível em: $<$ http://www.cancer.gov/cancerinfo/pdq/treatment/breast /HealthProfessional\#Section27>. Acesso em: $6 \mathrm{dez}$. 2007.

PRADO, M. A. S.; MAMEDE, M. V.; ALMEIDA, A. M.; CLAPIS, M. J. A prática da atividade física em mulheres submetidas à cirurgia por câncer de mama: percepção de barreiras e benefícios. Rev. Latino-am. Enfermagem. v. I2, n. 3, p. 494-502, 2004.

RICGI, M.D.; PINOTTI, J.A.; GARVALHO, F.M.; PINOTTI, M.; GIRIBELA, A.H.G. Influência da Preservação de Pele em Cirurgia Conservadora por Câncer de Mama sobre as Taxas de Recorrência Local e a Distância. RBGO. v. 25, n. 6, 2003.

ROSSI, L.; SANTOS, M.A. dos. Repercussões psicológicas do adoecimento e tratamento em mulheres acometidas pelo câncer de mama. Psicol. Gienc. Prof. v. 23, n. 4, dez. 2003.

SAKURADA, G. K.; TAQUEMORI, L. Y. Assistência Domiciliar. In: Cuidado Paliativo. Coordenação Institucional de Reinaldo Ayer de Oliveira. São Paulo: Conselho Regional de Medicina do Estado de São Paulo. p. I2O-I26, 2008.

SMELTZER, S.C.; BARE, B.G. Histórico e tratamento às pacientes com distúrbios mamários. In: Enfermagem médico-cirúrgica. Io ed. Rio de Janeiro, Guanabara Koogan, p. I529-I57I, 2004.

World Health Organization (WHO). Cancer. Fact sheet n.297. Feb. 2009. Geneva: World Health Organization, 2007. Disponível em: <http://www.who.int/mediacentre/factsheets/fs297/en/print.html $>$. 